\title{
¿LAS SOBRALIAS SE PUEDEN CLASIFICAR? - EL COMPLEJO DE SOBRALIA WARSZEWICZII
}

\author{
Robert L. DRESSLER \\ Jardín Botánico Lankester, Universidad de Costa Rica, P.O. Box 302-7050 Cartago, Costa Rica \\ robert.dressler@ucr.ac.cr
}

Resumen. Sobralia warszewiczii Rchb.f. fue descrita de Panamá occidental en 1852. El nombre se ha usado hasta el sur de México, aunque ninguna de las especies con flores lilas o moradas conocidas de Costa Rica concuerda del todo con S. warszewiczii. Una especie que forma colonias grandes en el noroeste de Costa Rica podría ser la misma especie que se encuentra en el sur de México, pero requiere más estudio, y no son nada claros los nombres correctos para los miembros de este complejo (salvo S. labiata Warsz. \& Rchb.f. y S. warszewiczii). Más al sur en Costa Rica parece que hay varias especies distintas y algunos enjambres híbridos.

AвSTRACt. Sobralia warszewiczii Rchb.f. Rchb.f. was described from western Panama in 1852. The name has been used as far away as southern Mexico, though, in fact, it is not definitely known from Costa Rica, where there are several species with lilac or purple flowers. A species that forms large colonies in northwestern Costa Rica may well be the same species that occurs in southern Mexico, but more study is needed, and it is not clear what the correct names are for any of this complex (except for S. labiata Warsz. \& Rchb.f. and $S$. warszewiczii). Farther south in Costa Rica, there appear to be several species and what are apparently hybrid swarms.

Palabras clave / Key words: Orchidaceae, clasificación, classification, Sobralia. S. warszewiczii, enjambres híbridos, hybrid swarms

En las últimas décadas, algunos colegas y su servidor hemos descrito aproximadamente 20 especies de Sobralia. Ahora tenemos algunas otras en preparación, y media docena que se pueden publicar tan pronto que haya material suficiente. Aquí, al contrario, quiero discutir algunas poblaciones a las cuales prefiero no aplicar nombres, por lo menos sin mucho más estudio. Las flores de Sobralia son sumamente delicadas, por lo cual es muy difícil identificar material prensado. A veces puede ser útil visitar las localidades típicas, donde los holotipos se colectaron, para ver si uno puede encontrar plantas que concuerden bien con las descripciones originales.

Las primeras especies de Sobralia de Costa Rica y Panamá fueron descritas por Reichenbach en 1852. La mayoría de ellas son nítidas y fáciles de reconocer, con la excepción notoria de S. bletiae Rchb.f. El tipo de $S$. bletiae tiene un pétalo ligeramente adherido al labelo en la base (afortunadamente, este holotipo se conserva muy bien). Reichenbach interpretó esto como un lóbulo lateral, y en su dibujo agregó otro igual al otro lado. Cuando vió la misma especie de nuevo, la describió como S. suaveolens Rchb.f.

Sobralia warszewiczii también fue descrita en 1852, y el nombre se ha usado desde Panamá hasta el sur de México. El Herbario Reichenbach contiene una muestra prensada más bien fea, probablemente preparada por Warszewicz en el campo. En 1866, Reichenbach publicó un dibujo de la flor, pero el dibujo muestra verrugas prominentes que no concuerdan muy bien con el ejemplar prensado. En esa ocasión Reichenbach escribió (en alemán) "Solamente en lugares húmedos en el Volcán Chiriquí" [ahora V. Barú], y "morada brillante." Afortunadamente, hemos encontrado plantas grandes de flores morado brillante a aproximadamente $2000 \mathrm{~m}$ de altura en el Volcán Barú, y confiamos que esta es la auténtica Sobralia warszewiczii. Hasta ahora, no hemos encontrado plantas de $S$. warszewiczii en Costa Rica, pero es muy probable que aparezcan, porque una de las poblaciones está muy cerca de la frontera tica.

¿Qué otras especies de flor lila hay en Costa Rica? 
Aparte de S. labiata que es muy distinta, dos supuestas especies se han descrito. Sobralia amparoae Schltr. fue colectada en el jardín de doña Amparo de Zeledón, que aparentemente estuvo hacia el lado norte de San José, pero no se sabe nada con respecto al origen de la planta. En el mismo artículo, Schlechter publicó Sobralia bradeorum Schltr., de "Costa Rica, San Jose, 1130 m, 1909." Es posible que aún hubieran sobralias silvestres en San José en 1909, o aún en 1923, cuando Schlechter publicó la especie. Ahora, las esperanzas de encontrar Sobralia silvestre en San José son aproximadamente iguales a las que hay de encontrar Cypripedium silvestre en Manhattan. Además, Schlechter notó que casi todas las flores de $S$. bradeorum fueron dañadas por pequeños insectos. Hay sobralias lilas de Panamá hasta Guatemala y el sur de México, pero parece que hay varias especies distintas, por lo menos en Costa Rica y Panamá.

Según Schlechter, el labelo de $S$. amparoae tenía cinco quillas, mientras que $S$. bradeorum tenía solamente tres. Con respecto a las quillas, el complejo de $S$. amparoae, $S$. bradeorum y $S$. warszewiczii, por lo general, tienen 3, 4 o 5 quillas principales, más una quilla más baja a cada lado. Me parece que hay variación dentro de las poblaciones. Por cierto, estas son las poblaciones que podrían representar enjambres híbridos. Otra característica a la que se ha dado mucha importancia es que plantas que crecen a mayor altura o en lugares muy expuestos tienen las hojas más bien coriáceas y fuertemente acanaladas, pero si se trasplanta una de estas plantas en un invernadero, estas características desaparecen.

En la provincia de Guanacaste hay poblaciones grandes de una Sobralia que demuestra mucha variación en color. Hay flores muy pálidas, otras con varios tonos de lila y algunas flores blancas, todas muy parecidas en estructura. Las poblaciones grandes de Guanacaste no muestran floración gregaria, sino que hay algunas pocas plantas en flor casi todos los días (en la época de floración). Las plantas de Guanacaste muestran el mismo comportamiento en cultivo. Más al sur, en la provincia de Alajuela, hay plantas más dispersas que parecen tener floración gregaria, pero la muestra aún es muy limitada. Al parecer, las poblaciones grandes de Guanacaste logran la polinización sin floración gregaria. De lo que he visto, las plantas de Guatemala y Chiapas bien podrían ser la misma especie que forma poblaciones grandes en Guanacaste, pero, otra vez, no hay aún una muestra adecuada.

En este momento, me parece que hay varias poblaciones de flor más o menos lila en Costa Rica (además de S. labiata). 1. Hay una especie que se extiende desde Coto Brus hasta el Valle de Orosí, en Cartago. Suponiendo que la planta de doña Amparo fue traída por su hermano de Turrialba, es muy posible que $S$. amparoae sería el nombre correcto para esta especie (Fig. 4). 2. Por la carretera al sur de Cartago, cerca de Cangreja (km. 30- 35) hay una Sobralia de flor más oscura, con algo de amarillo en el centro (Fig. 2). Uno ve las flores lila oscuro de vez en cuando por la carretera, pero muchas veces las flores están muy altas e inaccesibles. Otras veces, las condiciones del tránsito no permiten una parada rápida. Hemos colectado varias plantas sin flores, pero casi siempre resultan ser otras especies muy diferentes.

El año pasado, un amigo me llevó al Alto de Araya, a apenas unos $10 \mathrm{~km}$ al sureste del Jardín Botánico Lankester, pero hay que bajar al Valle de Orosí y subir por una de las peores carreteras que he visto en mi vida. En el Alto hay una laguna relativamente pequeña, pero llena de sobralias creciendo en los árboles y arbustos de la laguna. La variación en la laguna es tan grande que sugiere un enjambre híbrido, tal vez entre plantas de $S$. amparoae y otras muy oscuras con el centro pálido (Fig. 5). Don Abel Araya me dice que antes habían más plantas de flor oscura, pero son muy llamativas y al parecer los visitantes se han llevado casi todas las plantas de la laguna. Don Abel amablemente nos regaló una división de la planta en su jardín y está creciendo muy bien (ya tenemos plántulas en frasco.) Sería muy interesante hacer un estudio detallado de la población del Alto de Araya, pero la condición de la carretera no ayuda mucho. También hay lo que parece ser otro enjambre híbrido en La Laja, más al sur, también en Orosi. Afortunadamente, La Laja es de más fácil acceso, y creo que será mucho más factible hacer un estudio detallado con las plantas de La Laja. Las sobralias grandes, incluyendo las de flor lila, frecuentemente forman colonias grandes a los lados de carreteras nuevas, y en otros declives empinados y perturbados. Dodson (1998) sugirió que las especies $S$. ecuadorana Dodson, $S$. gentryi Dodson y S. powellii 


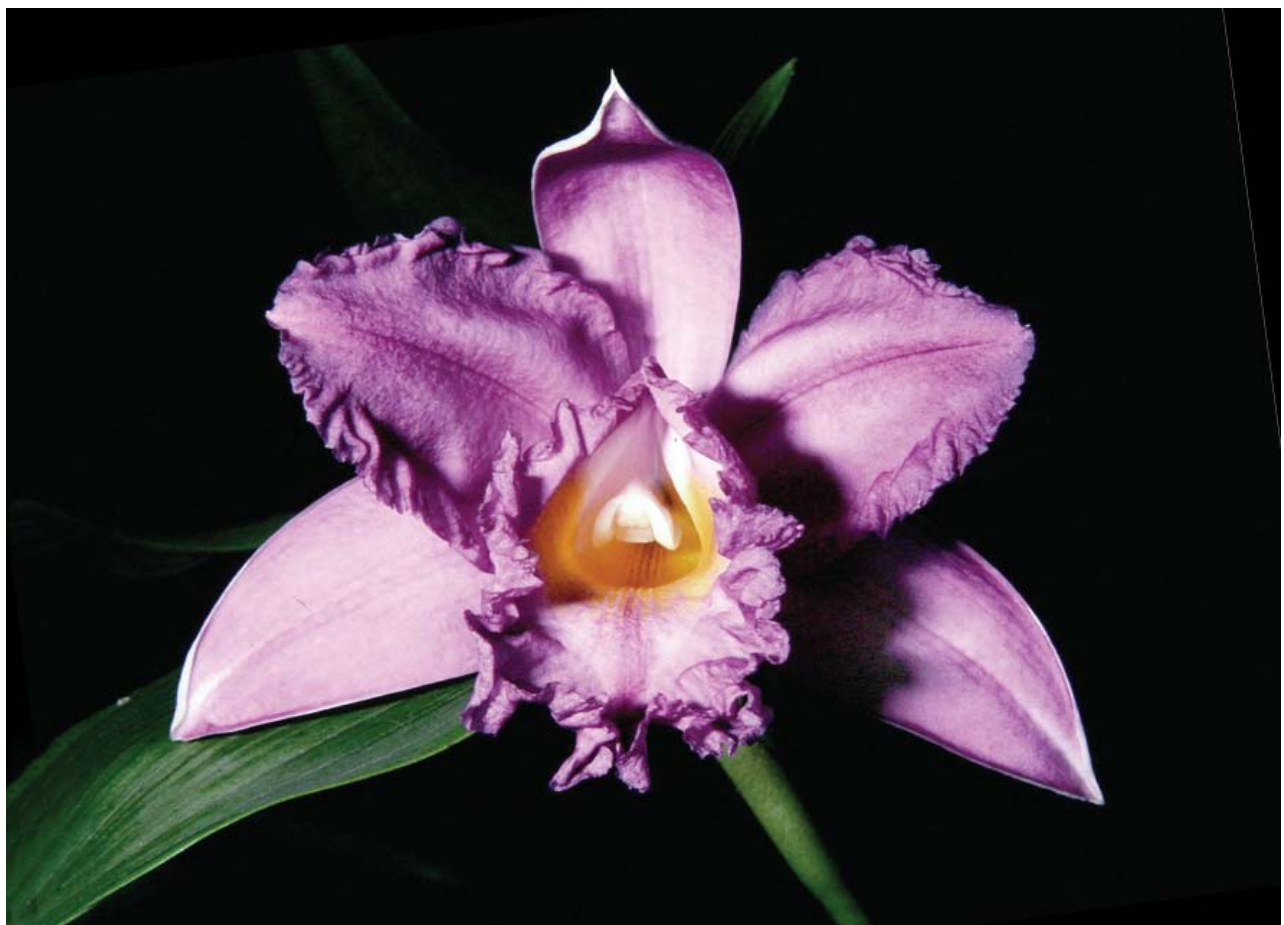

FIgure 1. Sobralia warszewiczii, una planta recolectada en el area de Río Sereno, Panamá, cerca de la frontera con Costa Rica, pero aún no hemos encontrado la especie en Costa Rica.

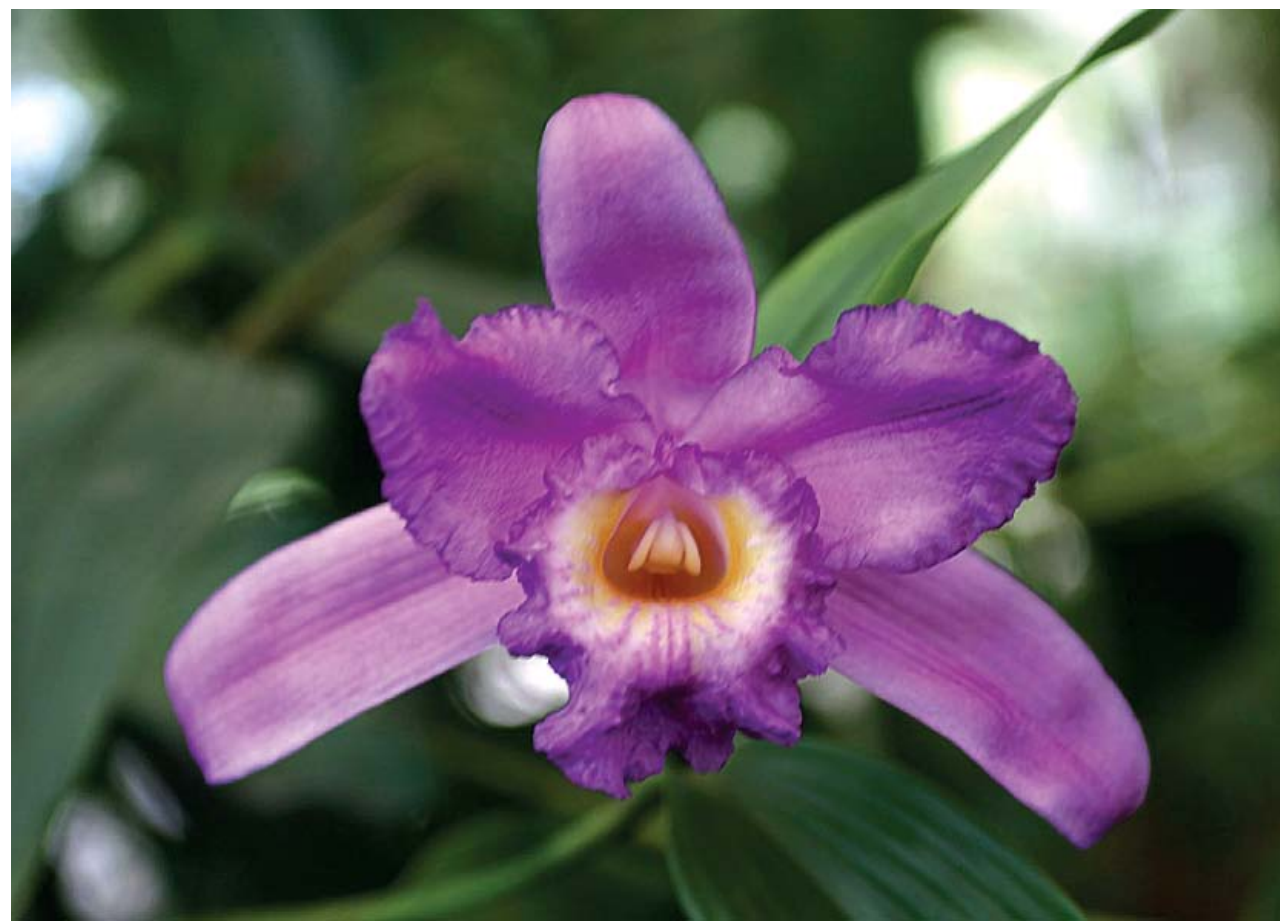

Figure 2. Sobralia sp., Esta especie se encuentra cerca de Cangreja, al sur de Cartago, Costa Rica. 


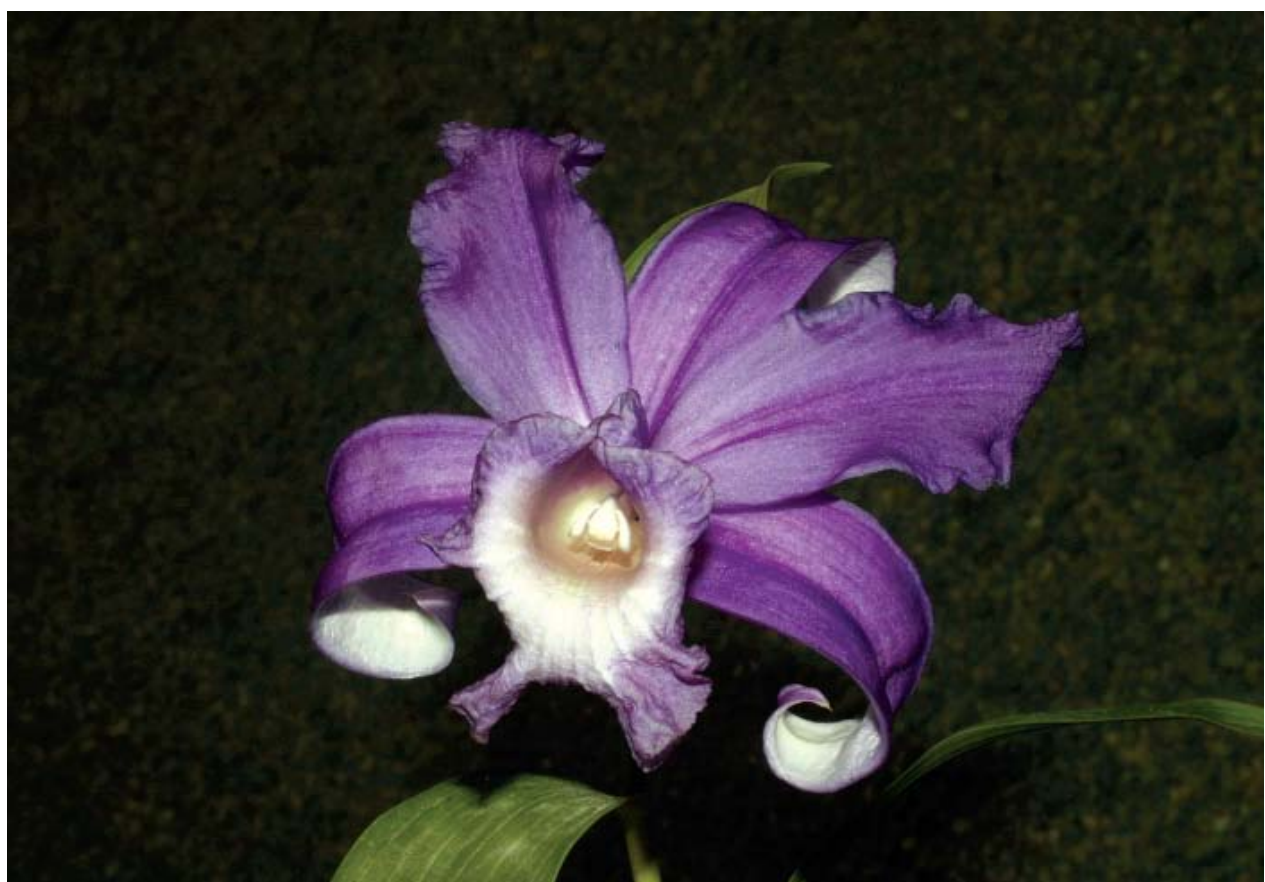

Figure 3. Sobralia sp. Alto de Araya (Cartago, Costa Rica); una división de la planta que crece en el jardín de don Abel Araya. Podría ser la única planta de esta especie que aún se encuentra cerca de la laguna.

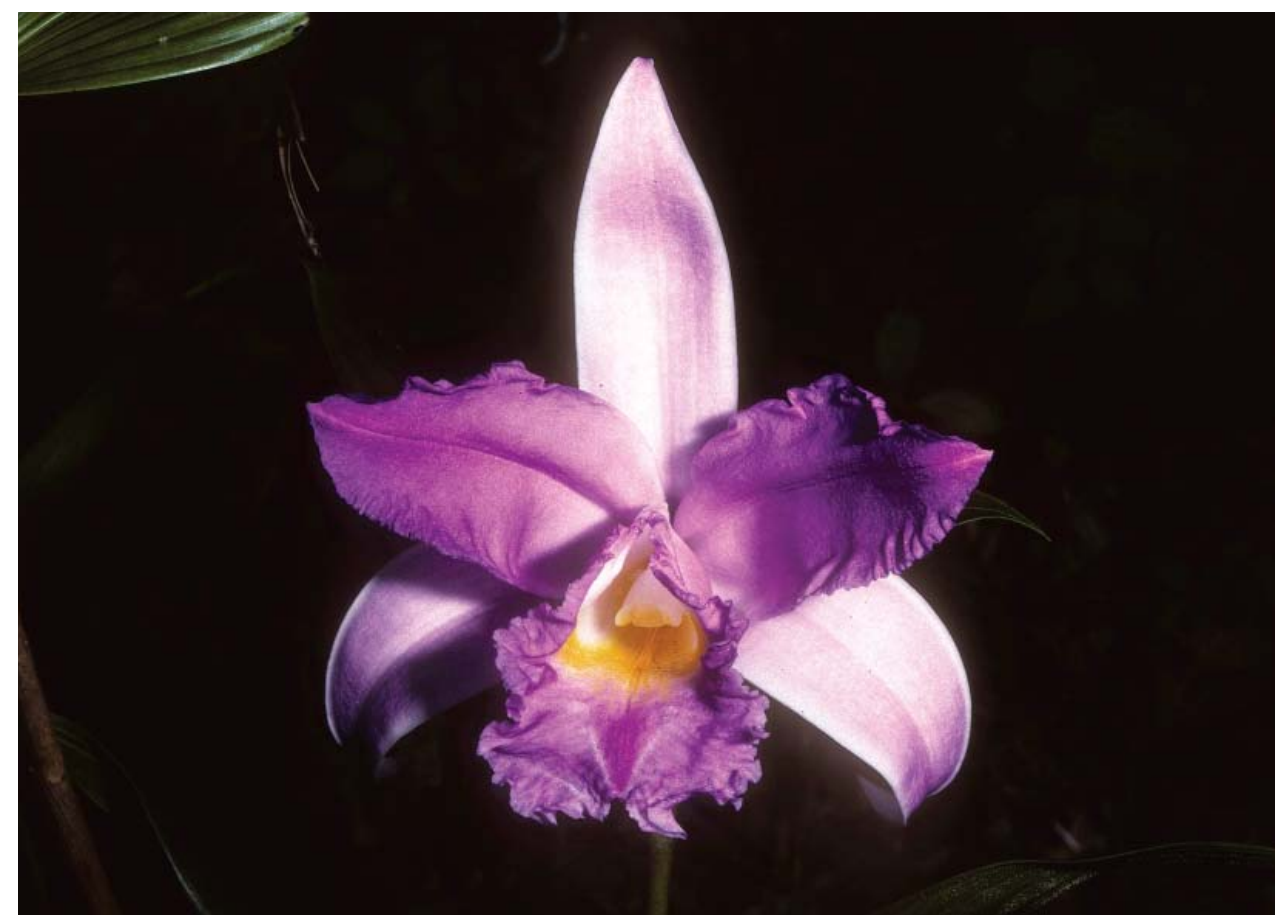

Figure 4. ¿Sobralia amparoae? Esta especie se encuentra de Coto Brus (Puntarenas, Costa Rica) hasta el área de Tapantí (Cartago, Costa Rica). Parece ser un elemento en los enjambres híbridos de La Laja y Alto de Araya. Aún no es claro cuales otras especies contribuyan genes a los enjambres híbridos. 


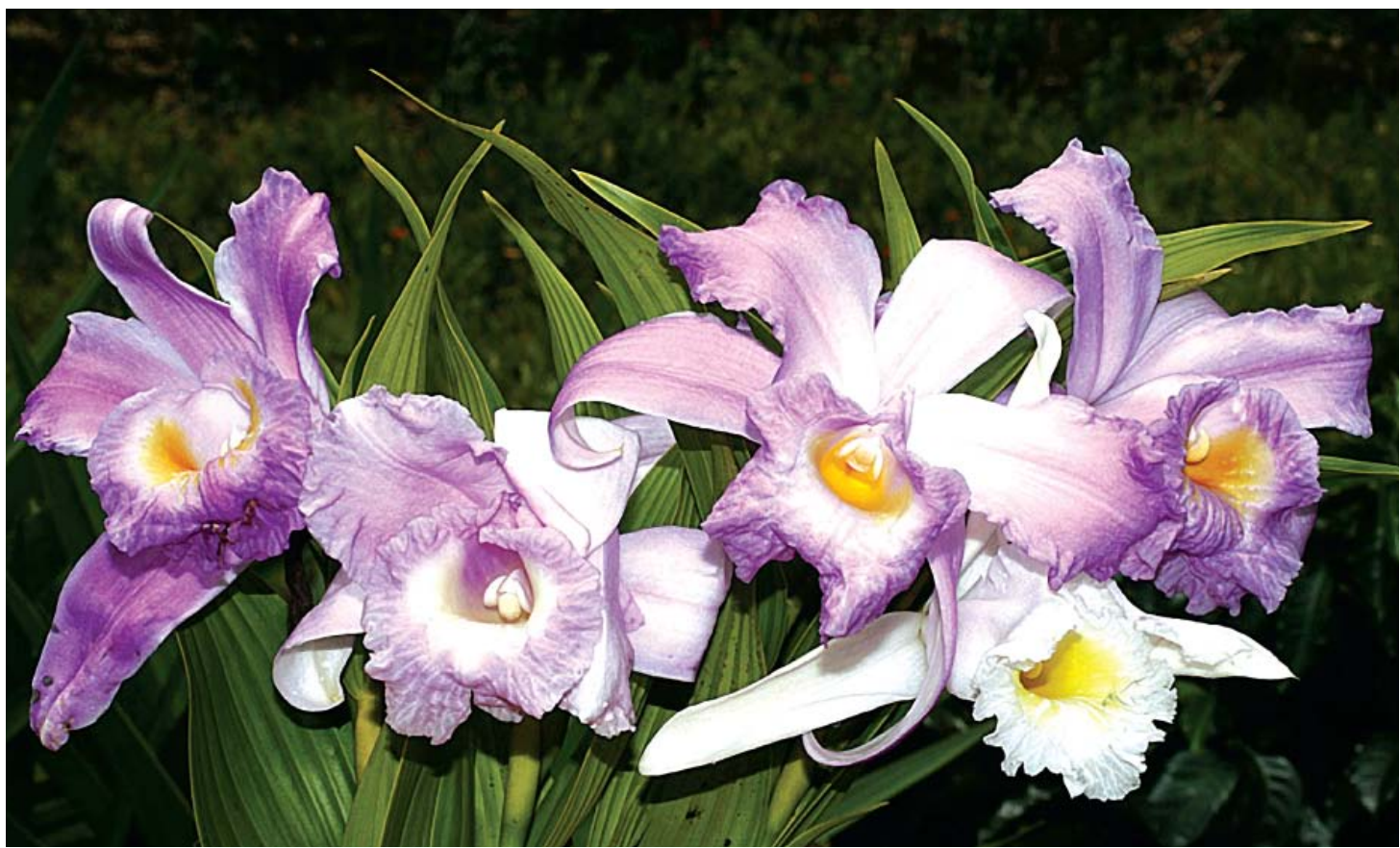

FIgURE 5. Una muestra de flores recolectadas por don Abel Araya en la laguna, Alto de Araya (Cartago, Costa Rica). La variación sugiere un enjambre híbrido.

Schltr. forman un enjambre híbrido a los lados de las carreteras, aunque las mismas especies no parecen formar híbridos en regiones no alteradas. Es muy probable que la "S. powellii" del Ecuador no tenga nada que ver con la que fue descrita de Panamá central, y hasta es posible que la "S. powellii" ecuatoriana sea una "especie" de origen híbrido.

\section{LiTERATURA CITADA}

Dodson, C. H. 1998. Nuevas especies y combinaciones del Ecuador. Fascículo 6. Orquideología 21: 3-67.

Reichenbach, H. G. 1852. Neue Orchideen der Expedition des Herrn J. de Warszewicz. Bot. Zeit. (Berlin) 10: 705715.

Reichenbach, H. G. 1866. Beitrage zu einer Orchideenkunde Central-Amerikas. Hamburg, Germany. 